\title{
Comprehensive Study of Ni Nanotubes for Bioapplications: From Synthesis to Payloads Attaching
}

\author{
A. L. Kozlovskiy, ${ }^{1,2}$ I. V. Korolkov, ${ }^{1,2}$ G. Kalkabay, ${ }^{3}$ M. A. Ibragimova, ${ }^{2}$ \\ A. D. Ibrayeva, ${ }^{1,2}$ M. V. Zdorovets, ${ }^{1,2,4,5}$ V. S. Mikulich, ${ }^{6}$ D. V. Yakimchuk, ${ }^{7}$ \\ A. E. Shumskaya, ${ }^{7}$ and E. Yu. Kaniukov ${ }^{7}$ \\ ${ }^{1}$ Eurasian National University, Astana, Kazakhstan \\ ${ }^{2}$ The Institute of Nuclear Physics of Republic of Kazakhstan, Astana, Kazakhstan \\ ${ }^{3}$ School of Engineering, Nazarbayev University, Astana, Kazakhstan \\ ${ }^{4}$ Ural Federal University Named after the First President of Russia B. N. Yeltsin, Yekaterinburg, Russia \\ ${ }^{5}$ National Research Nuclear University "MEPhI", Moscow, Russia \\ ${ }^{6}$ Institute of Chemistry of New Materials, National Academy of Science of Belarus, 36 Fr. Skaryna Street, 220141 Minsk, Belarus \\ ${ }^{7}$ Scientific and Practical Materials Research Centre, National Academy of Sciences of Belarus, 220072 Minsk, Belarus
}

Correspondence should be addressed to E. Yu. Kaniukov; ka.egor@mail.ru

Received 2 February 2017; Accepted 6 April 2017; Published 7 May 2017

Academic Editor: Renyun Zhang

Copyright (C) 2017 A. L. Kozlovskiy et al. This is an open access article distributed under the Creative Commons Attribution License, which permits unrestricted use, distribution, and reproduction in any medium, provided the original work is properly cited.

Due to the Ni nanotubes' shape anisotropy, low specific density, large specific surface, and uniform magnetic field, they have been offered as carriers for targeted delivery of drug or protein and the process of their formation from synthesis stage to the stage of surface modification and protein attaching has been demonstrated. Some steps to hasten their biomedical application have been applied. First, to have full control over the carrier dimensions and structure parameters, electrodeposition method in pores of polyethylene terephthalate template has been applied. Second, to understand the scope of Ni nanostructures application, their degradation in media with different acidity has been studied. Third, to improve the biocompatibility and to make payloads attachment possible, nanotubes surface modification with organosilicon compound has been carried out. At last, the scheme of protein attaching to the nanostructure surface has been developed and the binding process was demonstrated as an example of the bovine serum albumin.

\section{Background}

Biomedicine needs special chemical and physical techniques to rethink the approaches of treatment on the molecular and cellular scale. From this point of view magnetic nanostructures (NSs) are promising as a tool for prevention, diagnostics, and treatment of a wide range of diseases [1]. Control of shape, sizes, and chemical composition of NSs allows setting their physical properties at synthesis stage that opens a lot of opportunities for bioapplications, such as hyperthermia, cell separation [2], and biosensorics, as contrast substance in magnetic and sonoluminescence tomography $[3,4]$. The targeted delivery of payloads (drugs or proteins) by magnetic field is one of the quite interesting possibilities of NSs application. Drug or protein is associated with the magnetic NSs by functional groups, injected to circulatory system, and transported towards the problem area by magnetic field. Perspectives of this method have been shown in modeling studies $[5,6]$ and tests on animals $[7,8]$, but results of clinical tests have not been reported yet. It indicates that some difficulties are in the targeted delivery. One of them is selecting a carrier of the drug and protein delivery, which should have controllable magnetic properties and a low toxicity. The solution to this problem could be achieved by choosing the NSs shape and the suitable material.

In most cases, spherical magnetic nanoparticles are considered as carriers of drugs and proteins $[9,10]$. However, the small magnetic moment of these particles makes it difficult 
to focus magnetic field on them. This problem does not allow making a sufficient force for the blood flow resistance. Nanowires and nanotubes (NTs) allow overcoming typical limitations for nanoparticles due to their elongated form and anisotropy of magnetic properties [11-19]. In comparison with nanowires, NTs have some potential advantages. The absence of magnetic core makes it possible to create nanostructures with uniform magnetic field [15]. A lower density enables them to float in liquids (including the biological ones). The large specific surface area of NTs provides more functional groups to attach more cargo for the targeted delivery.

Considering the simplicity of physical properties modeling and the predictability of the behavior in biological media, preference is given to carriers designed on the base of pure magnetic materials (Fe, $\mathrm{Co}$, and $\mathrm{Ni}$ ), each of which has its advantages and disadvantages. The Fe NSs are often considered as the most attractive ones but could be easy oxidized in human liquids. Pure Co is more resistive but has a huge coercivity that leads to NSs conglomeration during their using in magnetic field. Ni NSs are also resistant to oxidation and have low coercivity that makes them the most attractive for the carrier creation. It should be noted that some nickel oxides are toxic and harmful to organic cells [20]. The problem of NSs oxidation could be solved by creation of inert shell (protective layer) on their surface. There are some requirements for protective layer such as absence of bioactivity, resistance to biodegradation, and stability, and it should have special functional groups to make links with payloads. These requirements satisfy organic (polymers) [21], inorganic (silane) [22], and gold coatings [23].

Considering that NTs based on ferromagnetic metals as carriers for targeted delivery have not been studied earlier, in our work a comprehensive investigation, from their synthesis process up to binding payloads, was carried out. The proposed method of NTs electrodeposition in PET template is simple and scalable, and it allows controlling the formed NSs parameters (length, diameter, and wall thickness) [24]. $\mathrm{Ni}$ has been selected as a NSs material, because it is a natural material and an essential nutrient [25]. Taking into account the toxicity of nickel oxides [20], Ni NTs degradation has been researched depending on the ambient medium acidity at different exposure times, and the opportunity of surface modification with organosilicon compound has been considered. Moreover, the scheme of protein attaching to the NSs surface was shown and bovine serum albumin binding was demonstrated as an example of payload.

\section{Methods}

Track-etched membranes based on polyethylene terephthalate (PET) were used as templates with a thickness of 12 microns, nominal pore diameter of $400 \mathrm{~nm}$, and a density of $4 \times 10^{7} \mathrm{~cm}^{-2}$. Electrochemical deposition was carried out at voltage of $1.75 \mathrm{~V}$ using the electrolyte: $\mathrm{NiSO}_{4} \times 6 \mathrm{H}_{2} \mathrm{O}$ $(100 \mathrm{~g} / \mathrm{l}), \mathrm{H}_{3} \mathrm{BO}_{3}(45 \mathrm{~g} / \mathrm{l})$, and ascorbic acid $(1.5 \mathrm{~g} / \mathrm{l})$ at temperature $25^{\circ} \mathrm{C} ; \mathrm{pH}$ of electrolyte was equal to 3 .

Characterization of structural features was conducted by scanning electron microscopy method (SEM, Hitachi
TM3030), Energy Dispersive X-ray Spectroscopy (EDS, Bruker XFlash MIN SVE), and X-ray diffraction analysis (XRD, Bruker D8 ADVANCE) using $\mathrm{Cu} \mathrm{K}_{\alpha}$ radiation and selected area electron diffraction (SAED, JEOL JEM-100). Control of internal diameters and estimation of wall thicknesses was conducted by methods of gas permeability (Sartocheck ${ }^{\circledR} 3$ Plus 16290) and transmission electron microscopy (TEM, JEOL JEM-100). Magnetic characteristics of Ni NTs were studied on universal measuring system (automated vibrating magnetometer) "Liquid Helium Free High Field Measurement System" (Cryogenic LTD) in magnetic fields $\pm 3 \mathrm{~T}$ at $300 \mathrm{~K}$ temperature.

To study Ni NTs reactivity in solutions with different $\mathrm{pH}$ value three aqueous solutions ranging from $\mathrm{pH}=1$ (strongly acidic) to 7 (neutral) were selected. Regulation of the solution acidity was carried out by adding a hydrochloric acid. NTs were kept in the solution with different $\mathrm{pH}$ over the time up to 20 days.

The amine functionalization of NTs surface was carried by adding $1 \mathrm{ml}$ of Ni NTs (3-aminopropyl) trimethoxysilane with concentration of 2 and $20 \mathrm{mM}$ in ethanol. The reaction mixture was placed in an ultrasound bath for 1-2 minutes and then NTs were kept in these solutions for 12 hours at room temperature. After the amine functionalization of Ni NTs, samples were washed in ethanol and dried at air.

The binding of the dye to the amino groups on the surface of the Ni NTs was carried out in $500 \mathrm{mM} / \mathrm{l}$ of Acid Orange dye solution (in $\mathrm{HCl}$ solution with $\mathrm{pH}=3$ ) for 12 hours. Samples were removed from solution after binding, twice washed with $\mathrm{HCl}$ solution $(\mathrm{pH}=3)$, and dried. Desorption of each sample was performed in $3 \mathrm{ml}$ of $\mathrm{NaOH}$ solution $(\mathrm{pH}=12)$ for 15 minutes on the shaker. The concentration of amino groups was determined from spectrophotometric measurements by the UV Specord 250 Plus Analytik Jena using the calibration graph. The measurement was carried out at the absorption maximum of Acid Orange dye, $495 \mathrm{~nm}$.

To bind protein (bovine serum albumin (BSA)) with the aminated surface, the NT powder was added to 500 microliters of acetate buffer $(\mathrm{pH}=4,7-4,8)$ and then to $100 \mu \mathrm{l}$ of $0.1 \mathrm{M}$ alcoholic solution of $\mathrm{N}$-(3-dimethylaminopropyl)$\mathrm{N}^{\prime}$-ethylcarbodiimide hydrochloride (EDC), $200 \mu \mathrm{l}$ of $0.1 \mathrm{M}$ solution of pentafluorophenol (PFP), and $100 \mu \mathrm{l}$ of BSA solution $(0.1 \mathrm{mg} / \mathrm{ml})$. The reaction flask was placed in the ultrasound bath for 1-2 minutes for better mixing NTs and further reacting for 8 hours at $24^{\circ} \mathrm{C}$. After reaction completion, samples were washed with ethanol and dried at $40-50^{\circ} \mathrm{C}$.

\section{Results and Discussion}

The nickel NTs were synthesized in pores of PET iontrack membranes by electrodeposition method. At deposition process, the cathode was a $10 \mathrm{~nm}$ thick gold film sputtered on back surface (not interacting with the electrolyte) of PET template. The thickness of the gold film kept open pores, and the NTs growth began at contact sites of gold with the electrolyte, as schematically shown in Figure 1(a). During sputtering process golden particles got inside pores and formed a golden ring around the pore. Going beyond the 


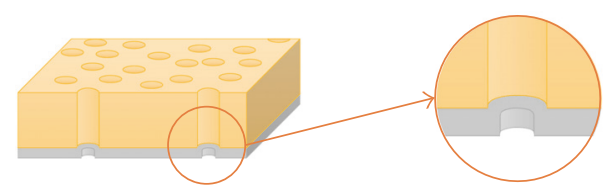

(a)

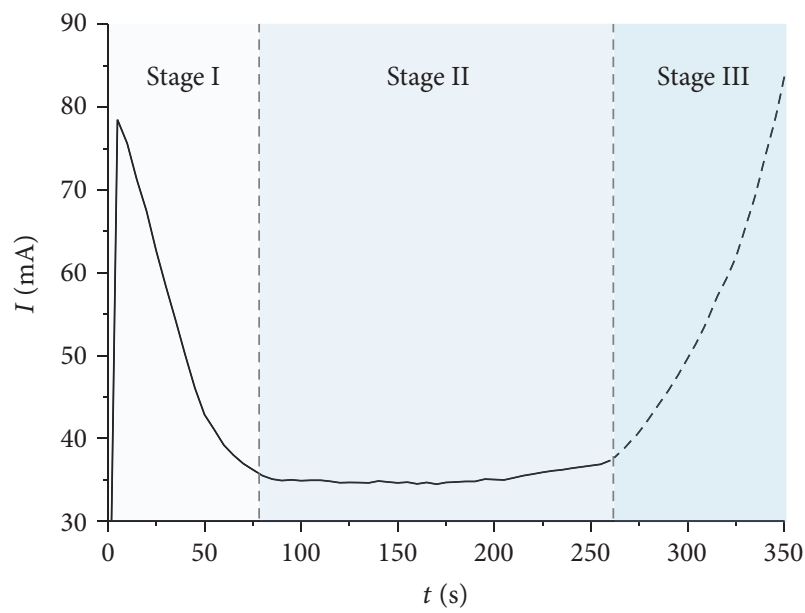

(b)

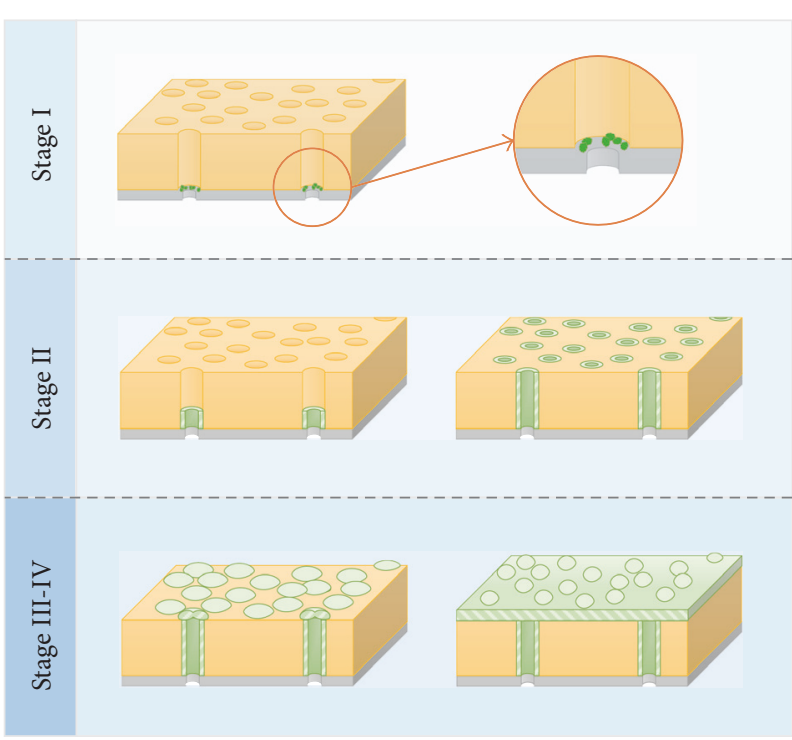

(c)

FIGURE 1: Schematic illustration of PET template with Au electrode for electrochemical formation of Ni NTs (a). Electrochemical deposition of $\mathrm{Ni}$ in pores of PET: chronoamperogram of deposition of Ni nanostructures from $\mathrm{NiSO}_{4} \times 6 \mathrm{H}_{2} \mathrm{O}(100 \mathrm{~g} / \mathrm{l}), \mathrm{H}_{3} \mathrm{BO}_{3}(45 \mathrm{~g} / \mathrm{l})$, and ascorbic acid $(1.5 \mathrm{~g} / \mathrm{l})$ electrolyte at potential $1.75 \mathrm{~V}$ (b) and schematic illustration of morphological dynamics of metallic phase at the key stages of synthesis.

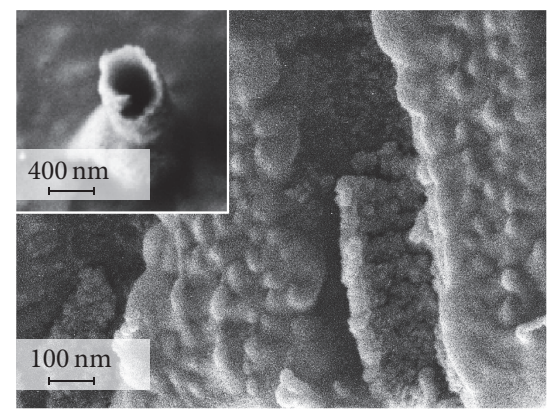

(a)

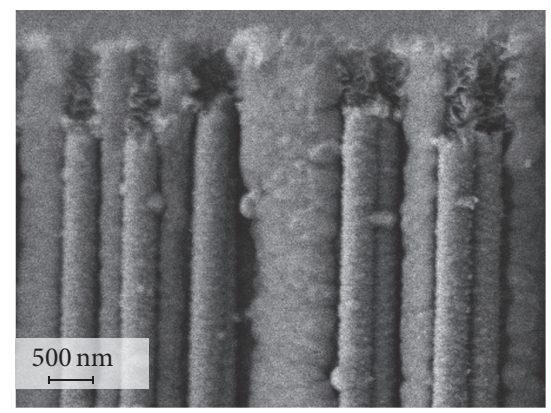

(b)

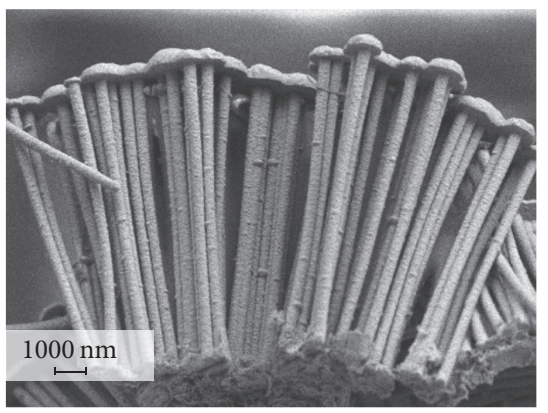

(c)

FIgURE 2: SEM images of Ni NTs formation at the first (a) with insert of individual tube, the second (b), and the third (c) stages.

pore edge the electrode defined the shape of formed nanostructures at the initial synthesis stages.

Figure 1(b) shows the characteristic time dependence values of the deposition current $(I)$ on time. In the beginning of the process $I$ reaches maximum value, after which it sharply drops and reaches a plateau. Constant $I$ value has been saved until 250 seconds, after which the deposition current growth occurs. This $I$ behavior can be explained by the fact that the process of electroplating metals into mask matrix pores involves the following four stages [26-31]: (I) the nucleation stage; (II) the stage of the intensive NTs growth; (III) the stage of the "caps" formation on the upper NTs part; (IV) the stage of the continuous metallic film formation on the mask surface (Figure 1(c)). SEM images corresponding to the (I)(III) deposition stages are shown in Figure 2.
It can be seen that the NTs formation occurs only at the two first stages of the electrodeposition process, which are shown in Figure 1(b). The first stage corresponds to the onset of the template pore filling. When the voltage is supplied, metal nuclei appear on the ring electrode in the bottom pore part, which replicates the electrode shape, forms a ring around golden cathode, and sets the tubular NSs shape (Figure 2(a)). Nuclei appear simultaneously in active zones on the surfaces of the pore walls, which contain defects, dangling bonds, and so on; these active zones are the result of chemical or physical activation, for example, in the course of pore formation in the ion-track-etched membranes and UV sensibilization [32]. The intensive nuclei formation reduces the concentration of metal ions inside the pore and, therefore, 


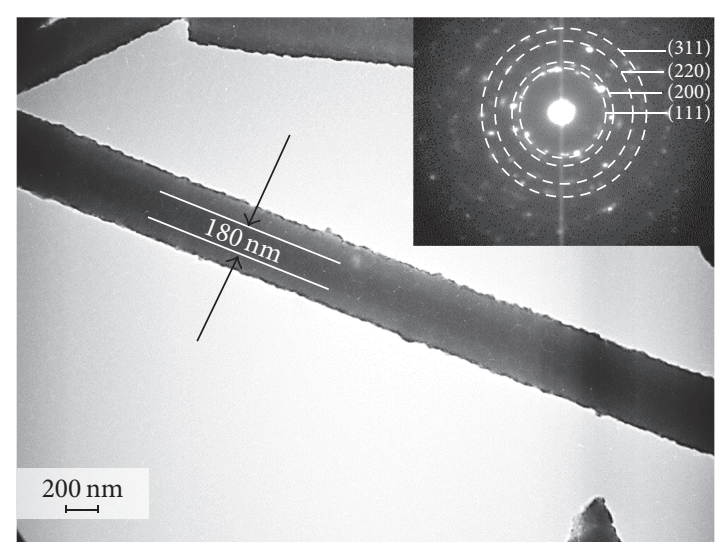

(a)

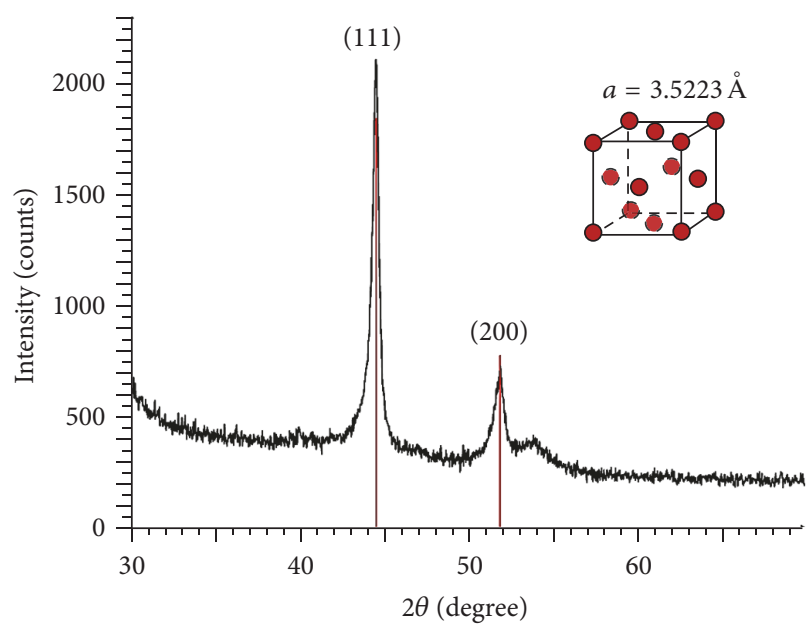

(b)

FIgURE 3: TEM image with SAED (inset to figure) (a) and XRD of Ni NTs (b).

leads to a substantial decrease of the deposition current (see Figure 1(b)).

At the second stage, I gradually decreases or remains nearly constant (Figure 1(b)), which corresponds to the NTs growth directly inside the pores (Figure 2(b)). This process has occurred until the NTs reach the template surface and "caps" form on the upper NTs part (Figure 2(c)). Since the ion diffusion into the pore occurs quite slowly, the NTs grow at the expense of the successive overlaying of twodimensional nuclei onto the ring part surface [33]. The deposition of two-dimensional layers leads to the formation of the crystal NTs structure. It should be noted that the influence of electrodeposition parameters on structural and morphological features of Ni NTs in detail is discussed in our work [24].

Analysis of SEM (Figure 2) and TEM images (Figure 3(a)) indicates that the formed NSs have a hollow shape and allow determining external diameters of Ni NTs equal to $400 \mathrm{~nm}$ over the entire length with deviations within $5-7 \%$ of the average values. The wall thickness could be estimated by TEM images $(\sim 120 \mathrm{~nm})$, but its resolution is not suitable for calibrating. That is why internal diameters of $180 \mathrm{~nm}$ were established by gas permeability method that corresponds to the wall thickness of $110 \mathrm{~nm}$. EDS spectra analysis has shown that NTs structure consists of pure nickel.

The crystal structure of Ni NTs was studied on the basis of the SAED analysis for individual NTs (insert in Figure 3(a)) and the X-ray diffraction spectra of NTs arrays into PET template (Figure 3(b)). NTs have preferred direction of the growth (111), which is also confirmed by the large ratio of the peaks (111) and (200) on the X-ray diffraction spectra. The XRD pattern recorded at diffraction angles $2 \theta<35^{\circ}$ and $2 \theta=53^{\circ}$ contains the peaks typical of PET film [34]. The XRD pattern contains the broadened peaks typical of diffraction on nanosized objects. The SAED analysis indicates a polycrystalline NTs structure. Studies of the X-ray spectra show that NTs have a face centered cubic (FCC) structure.

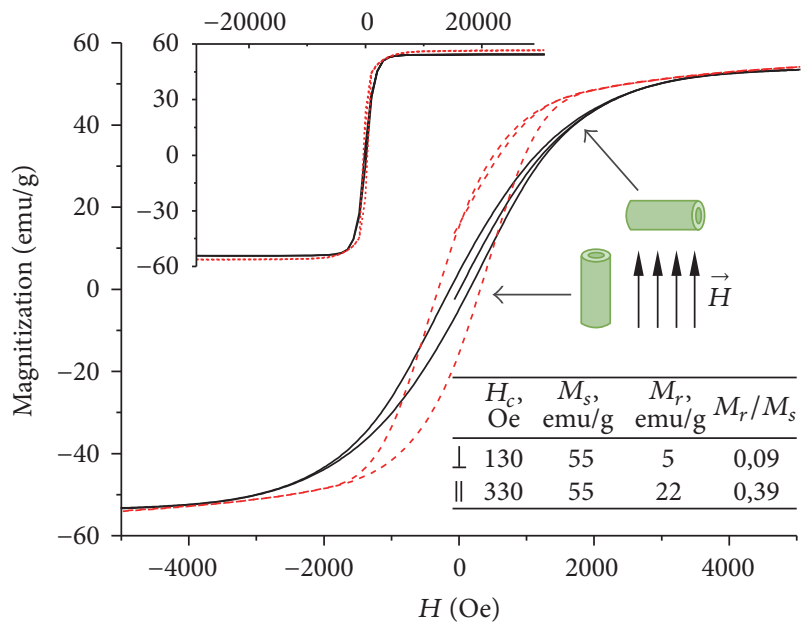

Figure 4: Hysteresis loop of Ni NTs arrays in PET template measured in magnetic field parallel (black solid line) and perpendicular (red dotted line) to the NTs axis at $300 \mathrm{~K}$.

The lattice parameter amounts $a=3.5223 \pm 0.0007 \AA$ and an average crystallite size is $25.3 \pm 1.1 \mathrm{~nm}$.

The degree of crystallite texturing that made up the NTs was determined by studying texture coefficients $T C(h k l)$, which were calculated using the Harris formula [35]. Calculations showed that $T C(h k l)$ at $2 \theta=44.559^{\circ}$ is equal to 1.5741 , and at $2 \theta=51.930^{\circ} \mathrm{TC}(\mathrm{hkl})=0.6741$. The values obtained for texture coefficients values indicate the presence of dominant direction [111] in the Ni NTs structure.

Measurements of the magnetization dependence on the magnetic field $M(H)$ were studied for parallel and perpendicular field directions with respect to orientation of the NTs axis (Figure 4). Based on hysteresis loops the main magnetic characteristics $\left(H_{\mathrm{c}}\right.$ : coercivity, $M_{\mathrm{r}}$ : remanent magnetization, $M_{\mathrm{s}}$ : magnetization of saturation, and $M_{\mathrm{r}} / M_{\mathrm{s}}$ : squareness ratio of hysteresis loop) were determined. 

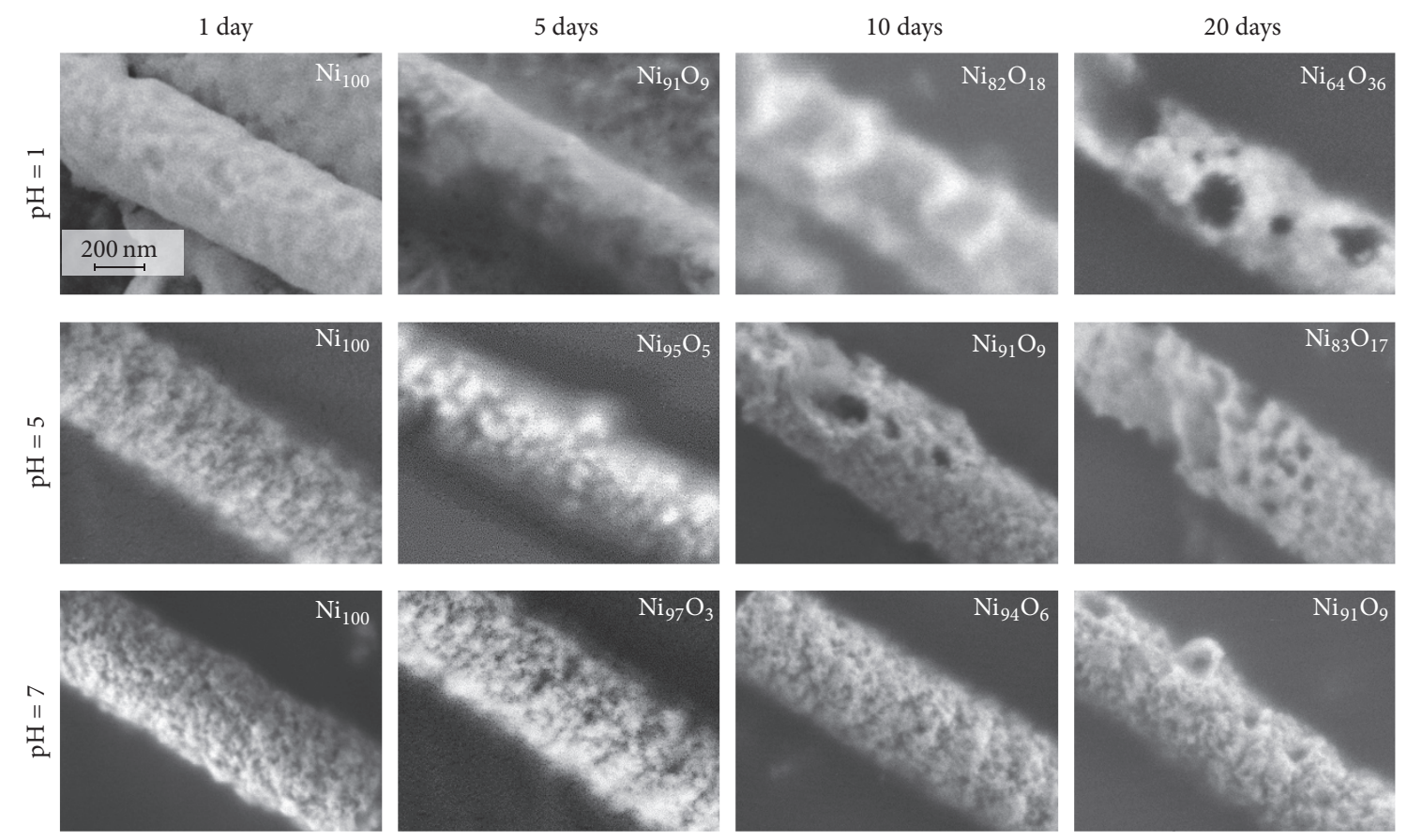

FIGURE 5: SEM images changes of Ni NTs morphology depending on acidity and the duration of exposure.

The study of Ni NTs arrays magnetization shows that stroke of hysteresis loop is similar to behavior of nanowires in magnetic field [36]. It should be noted that the loop measured at different directions of the magnetic field relative to the main axis of the NTs has different character, which indicates the presence of the magnetic anisotropy in the samples: coercivity and squarness value for parallel orientation $\left(H_{c||}=\right.$ 330 Oe, $M_{\mathrm{r}||} / M_{\mathrm{s} \|}=0.39$ ) of the field relative to the NTs axis is several times higher than the values for the perpendicular field direction $\left(H_{\mathrm{c} \perp}=130 \mathrm{Oe}, M_{\mathrm{r} \perp} / M_{\mathrm{s} \perp}=0.09\right)$. The anisotropy of the magnetic properties could be defined by either crystalline anisotropy, detected in the X-ray analysis, or shape anisotropy, caused by the fact that in an external magnetic field NTs with high aspect ratio ( 100) need higher energy of demagnetization along their axis than in the perpendicular direction of the field $[15,17]$.

It should be noted that two states are energetically favorable (stable) due to the absence of the magnetic core in NTs, when the magnetic field lines are directed along the tube axis, or when they are closed inside walls in the direction perpendicular to the axis. The homogeneity of NTs magnetic properties provides the behavior predictability in magnetic field that allows controlling direction and velocity of motion in liquids including biological ones. In addition, obtained nanostructures have a small remanent magnetization, which significantly reduces the probability of conglomerate formation of NTs, which are in a free state.

One of the most important characteristics of magnetic NTs for their application as magnetic carriers for targeted delivery is reactivity and rate of oxidation and degradation in environments with different $\mathrm{pH}$ value. Our studies were conducted in solutions with $\mathrm{pH}$ values of 1,5 , and 7 , which corresponds to the $\mathrm{pH}$ value of human body (fluid acidity of most organs in the human body is in the range $4-8$, except gastric acidity which is about 1 ). Thus, in simulated biological environment, we were able to get data about changes in Ni NTs structure over the time when they were within the human body. The changing in surface nanotube degradation was studied under the influence of environments with different acidity levels by both SEM and EDS analysis. The evaluation of morphology and composition of Ni NTs over the time up to 20 days is shown in Figure 5.

The largest degradation of NTs walls is observed for acidic media with $\mathrm{pH}=1$. On the tenth day with $\mathrm{pH}=1$ the formation of loose amorphous areas is observed on the outer side of nanotube walls consisting of oxide nickel compounds with the oxygen content of about $18 \%$. After 20 days the increasing area of amorphous regions and the increasing content of atomic oxygen in nanotube structure to $36 \%$ are observed, which lead to partial collapse of walls. Herewith EDS and XRD analysis has showed that formed amorphous regions consist of oxide compounds $\mathrm{NiO}$ and $\mathrm{Ni}_{2} \mathrm{O}_{3}$, which are toxic and harmful to organic cells, according to [20].

The oxygen appearance in structure on the fifth day for environments with $\mathrm{pH}=5$ and $\mathrm{pH}=7$ according to EDS is observed; however according to XRD oxide compounds in crystal structure appear on the tenth day. Small amount of oxygen impurities on the fifth day presents due to oxidation of NTs surface layer. On the twentieth day oxygen content in structure was $17 \%$ and $9 \%$ for $\mathrm{pH}=5$ and $\mathrm{pH}=7$, respectively. On the twentieth day for $\mathrm{pH}=5$ formation of amorphous regions is observed causing partial destruction of nanotube structure. For $\mathrm{pH}=7$ nodule formation is observed on tube surface, whose average size is $30-35 \mathrm{~nm}$ consisting of nickel oxide according to EDS and XRD. 


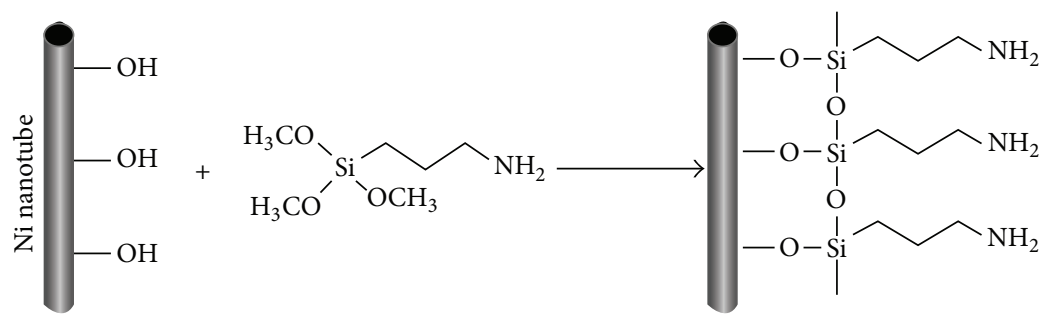

(a)

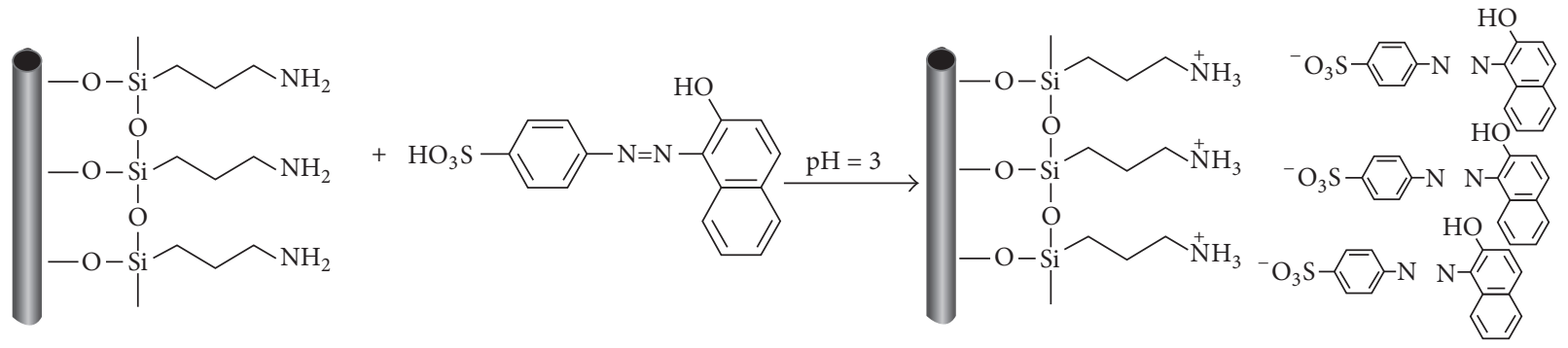

(b)

FIGURE 6: Schematic representation of amination of Ni NTs (a) and binding of Acid Orange dye to aminated surface (b).

Thus, based on the data obtained, we can prove that the long-term presence of Ni NSs (more than a day) in acidic media provokes deep degradation of NTs walls up to the destruction. Taking into account the fact that Ni oxides (II) and (III) are toxic to humans, NTs should be coated before using in biomedicine with resistance to biodegradation layers (e.g., gold, polymers, and silane). It should be noted that short, less than one day, processes (NTs extracting from templates and other types of treatment) were accompanied with slight structural changes on Ni NTs surface. Formation of nickel oxides on NTs surface makes its functionalization easier for the following payloads attaching.

Amine functionalization of the surface of Ni NTs was carried out by using organosilicon compound (3-aminopropyl)trimethoxysilane. The process is based on the chemical inertness of $\mathrm{Si}-\mathrm{C}$ linkages and the high reactivity of $\mathrm{Si}$ $\mathrm{O}$ bond which readily undergoes hydrolysis and reacted with $\mathrm{OH}$-terminated NTs surface. Organosilicon compounds have a low toxicity and $\mathrm{LD}_{50}$ can reach $5000 \mathrm{mg} / \mathrm{kg}$. Amination process is schematically shown in Figure 6(a).

After modification of the NTs with (3-aminopropyl)trimethoxysilane, the surface is coated by amine-terminated with high reactivity which is used to bind the protein thereto. For quantitative estimation of formed amino groups, colorimetric method using selective dye (Acid Orange) was employed, which was bound to the amino groups on the surface of the Ni NTs (Figure 6(b)). Colorimetric analysis results are shown in Table 1 . It is seen that the amination of a $20 \mathrm{mM}$ solution occurs with the highest yield.

To confirm the data of the colorimetric analysis, the samples modified with organosilicon compound were examined by SEM with EDS mapping of the surface (Figure 7). In the SEM image (Figure 7(a)), a change of the NTs surface with respect to the uncoated sample shown in Figure 2 is considerably different. EDS mapping (Figures 7(b) and 7(c))
TABLE 1: The concentration of amino groups on the surface of $\mathrm{Ni}$ NTs.

\begin{tabular}{|c|c|}
\hline Sample & {$\left[\mathrm{NH}_{2}\right], \mu \mathrm{M} / \mathrm{g}$} \\
\hline Before modification & 0.05 \\
\hline NT-NH ${ }_{2}(2 \mathrm{mM})$ & 4.25 \\
\hline NT-NH ${ }_{2}(20 \mathrm{mM})$ & 7.39 \\
\hline
\end{tabular}

clearly shows that the organosilicon compound is localized exceptionally in the surface of the NTs.

The binding protein bovine serum albumin (BSA) was performed with the aminated surface through the activated carboxyl groups. The process is schematically shown in Figure 8 .

Considering that FT-IR spectroscopy is a powerful method for identification and investigation of a secondary protein structure, modified samples were studied by infrared spectroscopy using the setup for FT-IR attenuated total reflectance (ATR) spectroscopy. FT-IR spectra of pure Ni NTs (blue), BSA (red), and NTs after modification by BSA protein (black) are shown in Figure 9.

On the FT-IR spectra of Ni NTs modified by BSA protein in the region $1000-3500 \mathrm{~cm}^{-1}$ new behavior of the curve is observed compared with the pure FT-IR spectra of NTs: appearance of new peaks at $3100-3400 \mathrm{~cm}^{-1}$ (associated $\mathrm{NH}$ group), $1650 \mathrm{~cm}^{-1}$ ( $\mathrm{C}=\mathrm{O}$ peptides), $1540 \mathrm{~cm}^{-1}$ (C-N peptides bond), and $1450 \mathrm{~cm}^{-1}$ (terminal ionized COO-groups) related to the BSA protein which was covalent-bonded with NTs surface. The results indicate that proteins could be attached to the modified by amination Ni NTs. This indicates the ability to bind complex molecules to NTs that can be hereinafter used in the targeted delivery of drugs and proteins. 


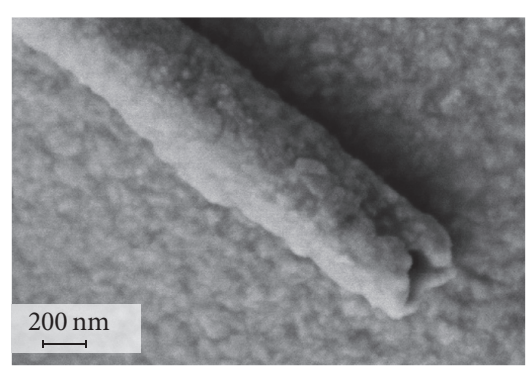

(a)

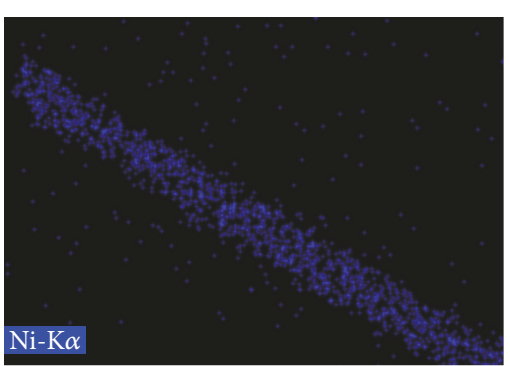

(b)

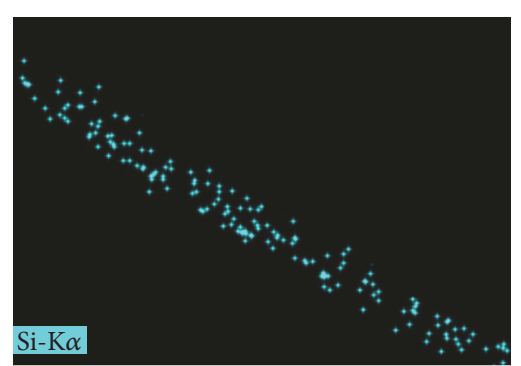

(c)

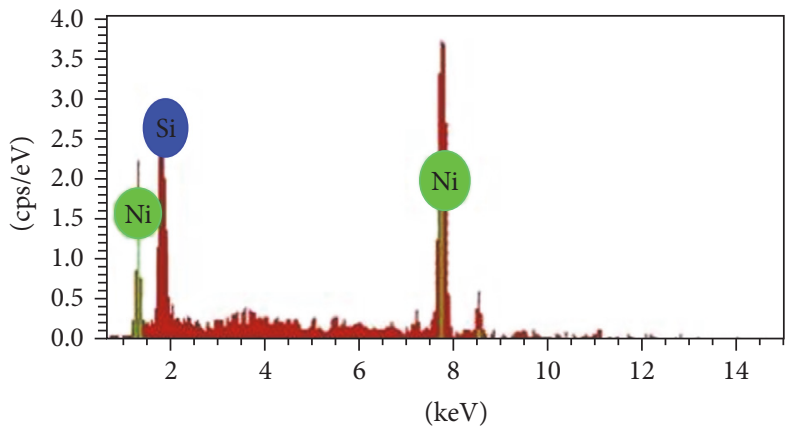

(d)

FIGURE 7: SEM image of NTs surface modified with organosilicon compound (a); EDS mapping of the NTs surface at the nickel (b) and silicon (c) detection mode; EDS spectra (d).<smiles>CCN=C=NCCC[NH+](C)Cl</smiles>

(a)<smiles>CO[Si](C)(CCCN)O[Si](CCCN)(OC)O[Si](C)(C)CCCN</smiles><smiles>CO[Si](C)(CCCNC(=O)S)O[Si](CCCNC(=O)CS)(OC)O[Si](C)(CCCNC(=O)CS)OC</smiles>

(b)

FIGURE 8: Scheme protein interaction with the surface of the aminated NTs.

\section{Conclusions}

Comprehensive study of Ni NTs for bioapplications from synthesis to payloads attaching stages was carried out. The step-by-step growth mechanism of NTs at electrodeposition process in polyethylene terephthalate template was analyzed and possibility of formation of nanostructures with tunable parameters by adapting the deposition parameters and template dimensions was shown. Quite large magnetization at low magnetic fields and magnetic anisotropy of Ni NTs provide their predictable behavior at the movement in the flow of biological fluids. The investigation of degradation degree 


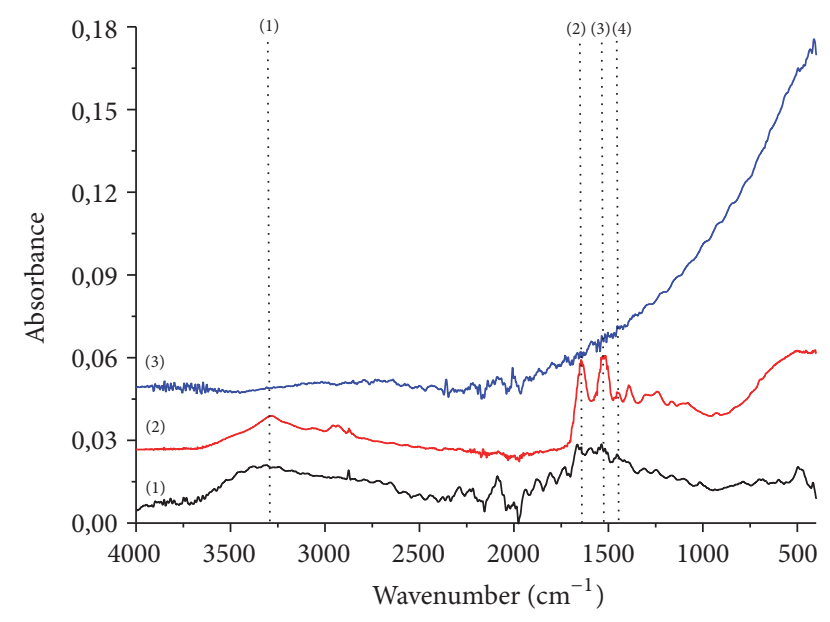

FIGURE 9: FT-IR spectrum of BSA attached to the surface of Ni NTs (1), BSA (2), and Ni NTs (3).

of Ni NTs in environments with $\mathrm{pH}$ close to the human liquids has shown a significant effect of the medium acidity on the NTs oxidation rate up to the destruction of their structure. Moreover, the oxidation leads to formation of oxide compounds $\mathrm{NiO}$ and $\mathrm{Ni}_{2} \mathrm{O}_{3}$ on the nanostructures surfaces, which are toxic and harmful to organic cells. On the other hand, Ni NTs can be functionalized by organosilicon compounds with a low toxicity. The possibility of surface functionalization with (3-aminopropyl)trimethoxysilane, as well as using of functionalized NTs in proteins attaching, has been demonstrated as an example of the binding bovine serum albumin with the aminated surface through the activated carboxyl groups. This investigation demonstrates the prospects of Ni NTs for bioapplication, for instance, to the targeted drug delivery of drags or proteins by means of a magnetic field.

\section{Conflicts of Interest}

The authors declare that they have no conflicts of interest.

\section{References}

[1] S. Bucak, B. Yavuztürk, and A. D. Sezer, "Magnetic nanoparticles: synthesis, surface modifications and application in drug delivery," in Recent Advances in Novel Drug Carrier Systems, vol. 2, pp. 165-200, InTech, Rijeka, Croatia, 2012.

[2] D. T. Nguyen and K. Kim, "Functionalization of magnetic nanoparticles for biomedical applications," Korean Journal of Chemical Engineering, vol. 31, no. 8, pp. 1289-1305, 2014.

[3] S. K. Yen, P. Padmanabhan, and S. T. Selvan, "Multifunctional iron oxide nanoparticles for diagnostics, therapy and macromolecule delivery," Theranostics, vol. 3, no. 12, pp. 986-1003, 2013.

[4] N. Lee and T. Hyeon, "Designed synthesis of uniformly sized iron oxide nanoparticles for efficient magnetic resonance imaging contrast agents," Chemical Society Reviews, vol. 41, no. 7, pp. 2575-2589, 2012.

[5] C. Alexiou, W. Arnold, R. J. Klein et al., "Locoregional cancer treatment with magnetic drug targeting," Cancer Research, vol. 60, no. 23, pp. 6641-6648, 2000.
[6] S. Goodwin, C. Peterson, C. Hoh, and C. Bittner, "Targeting and retention of magnetic targeted carriers (MTCs) enhancing intra-arterial chemotherapy," Journal of Magnetism and Magnetic Materials, vol. 194, no. 1, pp. 132-139, 1999.

[7] Y. Hirota, Y. Akiyama, Y. Izumi et al., "Fundamental study for development magnetic drug delivery system," Physica C: Superconductivity, vol. 469, no. 15-20, pp. 1853-1856, 2009.

[8] Y. Yoshida, S. Fukui, S. Fujimoto et al., "Ex vivo investigation of magnetically targeted drug delivery system," Journal of Magnetism and Magnetic Materials, vol. 310, no. 2, pp. 28802882, 2007.

[9] S. R. Dave and X. Gao, "Monodisperse magnetic nanoparticles for biodetection, imaging, and drug delivery: A versatile and evolving technology," Wiley Interdisciplinary Reviews: Nanomedicine and Nanobiotechnology, vol. 1, no. 6, pp. 583-609, 2009.

[10] S. Mura, J. Nicolas, and P. Couvreur, "Stimuli-responsive nanocarriers for drug delivery," Nature Materials, vol. 12, no. 11, pp. 991-1003, 2013.

[11] A. Prina-Mello, Z. Diao, and J. M. D. Coey, "Internalization of ferromagnetic nanowires by different living cells," Journal of Nanobiotechnology, vol. 4, no. 1, 9 pages, 2006.

[12] X. Zhang, H. Zhang, T. Wu et al., "Comparative study in fabrication and magnetic properties of FeNi alloy nanowires and nanotubes," Journal of Magnetism and Magnetic Materials, vol. 331, pp. 162-167, 2013.

[13] L. Zhang, T. Petit, K. E. Peyer, and B. J. Nelson, “Targeted cargo delivery using a rotating nickel nanowire," Nanomedicine: Nanotechnology, Biology, and Medicine, vol. 8, no. 7, pp. 1074-1080, 2012.

[14] K. M. Pondman, N. D. Bunt, A. W. Maijenburg et al., "Magnetic drug delivery with FePd nanowires," Journal of Magnetism and Magnetic Materials, vol. 380, pp. 299-306, 2015.

[15] T. N. Narayanan, M. M. Shaijumon, P. M. Ajayan, and M. R. Anantharaman, "Synthesis of high coercivity core-shell nanorods based on Nickel and Cobalt and their magnetic properties," Nanoscale Research Letters, vol. 5, no. 1, pp. 164-168, 2010.

[16] S. Shamaila, D. P. Liu, R. Sharif et al., "Electrochemical fabrication and magnetization properties of CoCrPt nanowires and nanotubes," Applied Physics Letters, vol. 94, Article ID 203101, 2009.

[17] R. Sharif, S. Shamaila, M. Ma et al., "Magnetic switching of ferromagnetic nanotubes," Applied Physics Letters, vol. 92, no. 3, Article ID 032505, 2008.

[18] R. Sharif, S. Shamaila, M. Ma et al., "Magnetic and microstructural characterizations of CoFe and CoFeB nanowires," Journal of Magnetism and Magnetic Materials, vol. 320, no. 8, pp. 15121516, 2008.

[19] A. E. Shumskaya, E. Y. Kaniukov, A. L. Kozlovskiy et al., "Structure and physical properties of iron nanotubes obtained by template synthesis," Physics of the Solid State, vol. 59, no. 4, pp. 766-772, 2017.

[20] K. Lascelles, L. G. Morgan, D. Nicholls et al., "Nickel compounds," in Ullmann's Encyclopedia of Industrial Chemistry, Wiley-VCH Verlag GmbH \& Co. KGaA, Weinheim, Germany, 2000.

[21] J. R. Smith and D. A. Lamprou, "Polymer coatings for biomedical applications: a review," Transactions of the IMF, vol. 92, no. 1, pp. 9-19, 2014.

[22] S. R. Torati, V. Reddy, S. S. Yoon, and C. G. Kim, "Protein immobilization onto electrochemically synthesized CoFe nanowires," 
International Journal of Nanomedicine, vol. 10, pp. 645-651, 2015.

[23] K. M. Pondman, A. W. Maijenburg, F. B. Celikkol et al., "Au coated Ni nanowires with tuneable dimensions for biomedical applications," Journal of Materials Chemistry B, vol. 1, no. 44, pp. 6129-6136, 2013.

[24] A. L. Kozlovskiy, D. I. Shlimas, A. E. Shumskaya et al., "Influence of electrodeposition parameters on structural and morphological features of Ni nanotubes," Physics of Metals and Metallography, vol. 118, no. 2, pp. 174-179, 2017.

[25] N. W. Solomons, F. Viteri, T. R. Shuler, and F. H. Nielsen, "Bioavailability of nickel in man: Effects of foods and chemicallydefined dietary constituents on the absorption of inorganic nickel," Journal of Nutrition, vol. 112, no. 1, pp. 39-50, 1982.

[26] B. Yoo, F. Xiao, K. N. Bozhilov, J. Herman, M. A. Ryan, and N. V. Myung, "Electrodeposition of thermoelectric superlattice nanowires," Advanced Materials, vol. 19, no. 2, pp. 296-299, 2007.

[27] M. Motoyama, Y. Fukunaka, T. Sakka, and Y. H. Ogata, "Initial stages of electrodeposition of metal nanowires in nanoporous templates," Electrochimica Acta, vol. 53, no. 1, pp. 205-212, 2007.

[28] M. P. Proenca, C. T. Sousa, J. Ventura et al., "Distinguishing nanowire and nanotube formation by the deposition current transients," Nanoscale Research Letters, vol. 7, no. 1, p. 280, 2012.

[29] X. F. Han, S. Shamaila, R. Sharif et al., "Structural and magnetic properties of various ferromagnetic nanotubes," Advanced Materials, vol. 21, no. 45, pp. 4619-4624, 2009.

[30] T. N. Narayanan, M. M. Shaijumon, L. Ci, P. M. Ajayan, and M. R. Anantharaman, "On the growth mechanism of nickel and cobalt nanowires and comparison of their magnetic properties," Nano Research, vol. 1, no. 6, pp. 465-473, 2008.

[31] E. Y. Kaniukov, A. L. Kozlovsky, D. I. Shlimas et al., "Electrochemically deposited copper nanotubes," Journal of Surface Investigation: X-ray, Synchrotron and Neutron Techniques, vol. 11, no. 1, pp. 270-275, 2017.

[32] B. Bercu, I. Enculescu, and R. Spohr, "Copper tubes prepared by electroless deposition in ion track templates," Nuclear Instruments and Methods in Physics Research, Section B: Beam Interactions with Materials and Atoms, vol. 225, no. 4, pp. 497502, 2004.

[33] L. M. Graham, S. Cho, S. K. Kim, M. Noked, and S. B. Lee, "Role of boric acid in nickel nanotube electrodeposition: a surfacedirected growth mechanism," Chemical Communications, vol. 50, no. 5, pp. 527-529, 2014.

[34] E. Kaniukov, A. Kozlovsky, D. Shlimas et al., "Tunable synthesis of copper nanotubes," in Proceedings of the IOP Conference Series: Materials Science and Engineering, vol. 110, p. 012013, Tomsk, Russia, 2016.

[35] J. I. Langford and A. J. Wilson, "Scherrer after sixty years: a survey and some new results in the determination of crystallite size," Journal of Applied Crystallography, vol. 11, no. 2, pp. 102113, 1978.

[36] G. C. Han, B. Y. Zong, P. Luo, and Y. H. Wu, "Angular dependence of the coercivity and remanence of ferromagnetic nanowire arrays," Journal of Applied Physics, vol. 93, no. 11, pp. 92029207, 2003. 

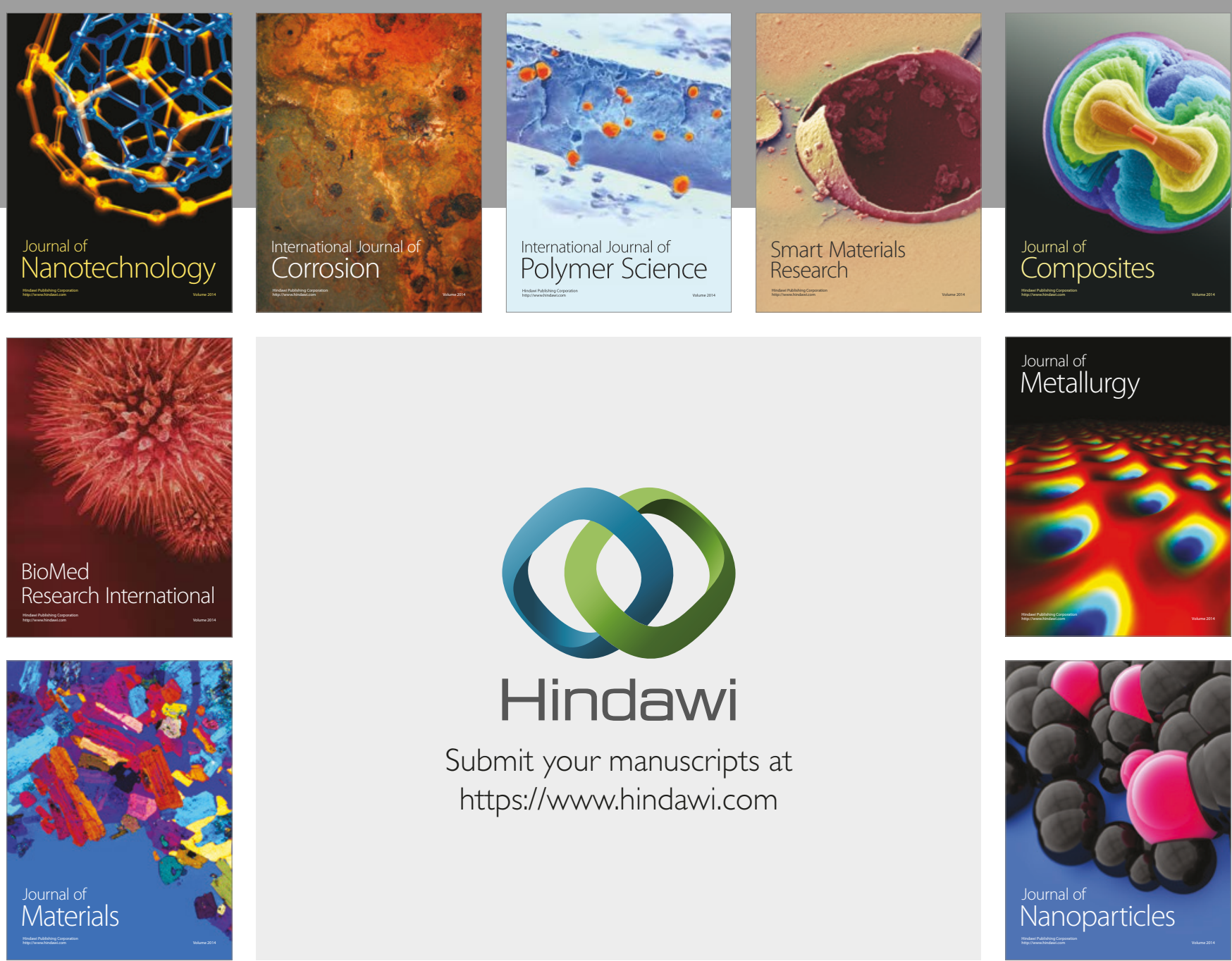

\section{Hindawi}

Submit your manuscripts at

https://www.hindawi.com
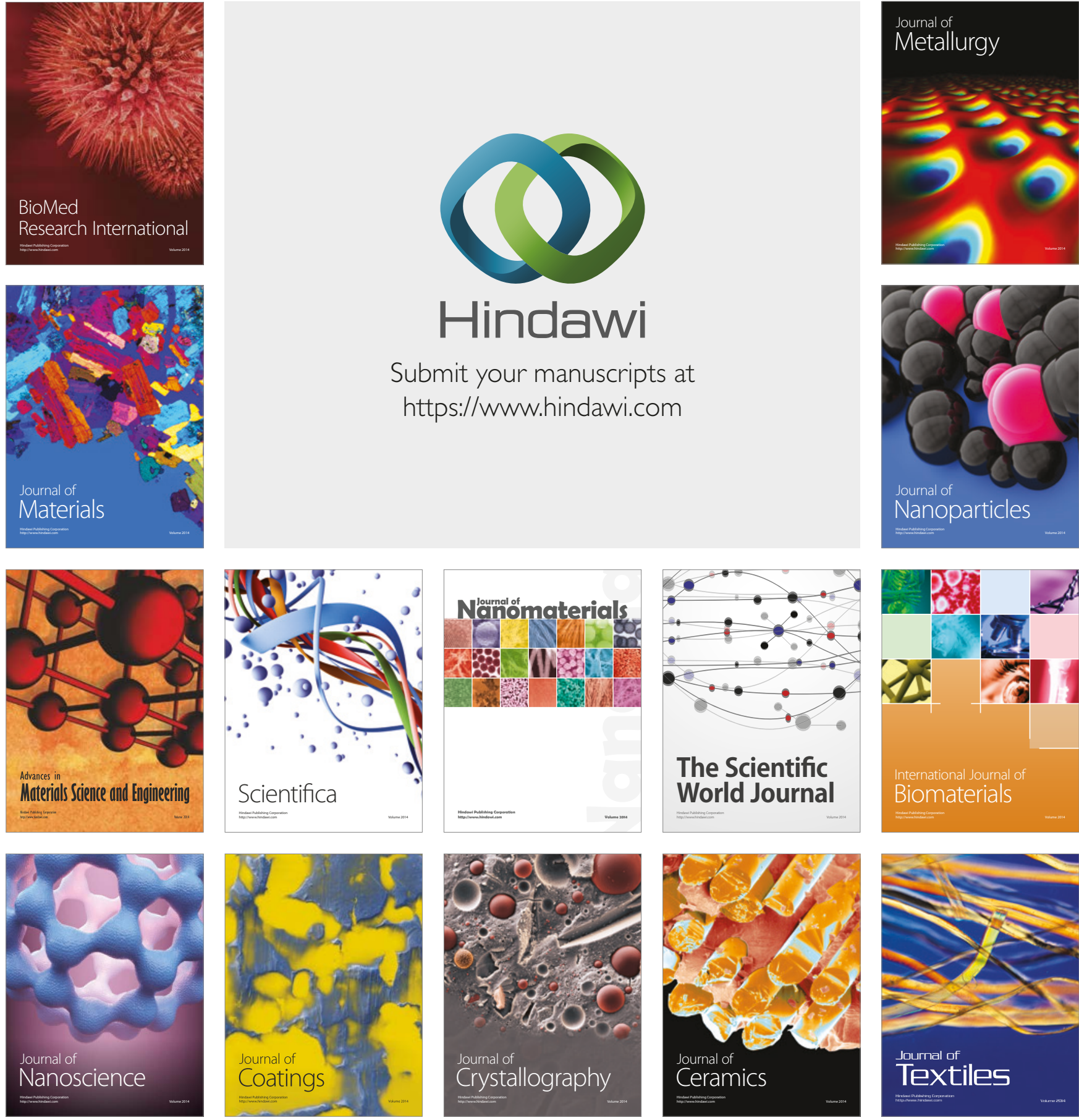

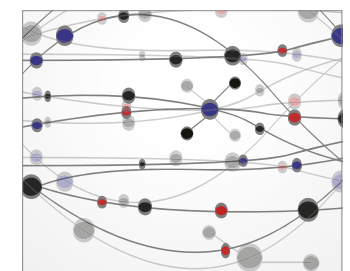

The Scientific World Journal
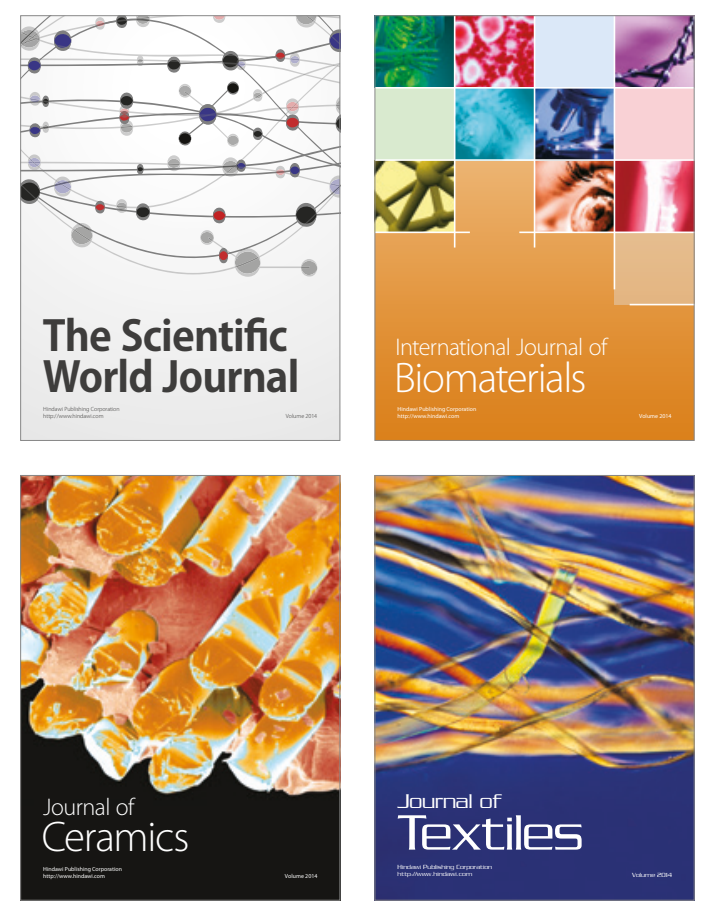